\title{
Clones of Self-Dual and Self-K-Al Functions in K-valued Logic
}

\author{
M. A. Malkov \\ Russian Research Center for Artificial Intelligence, Moscow, Russia
}

\section{Email address:}

mamalkov@gmail.com

\section{To cite this article:}

M. A. Malkov. Clones of Self-Dual and Self-K-Al Functions in K-valued Logic. Pure and Applied Mathematics Journal. Vol. 6, No. 2, 2017, pp. 59-70. doi: 10.11648/j.pamj.20170602.11

Received: February 17, 2017; Accepted: February 14, 2017; Published: March 10, 2017

\begin{abstract}
We give a classification of dual functions, they are $m$-al functions. We call a function $m$-al with respect to an operator if the operator lives any function unchanged after $m$ times of using the operator. And $2 \leq \mathrm{m} \leq \mathrm{k}$. Functions with different $m$ have very different properties. We give theoretical results for clones of self-dual $(\mathrm{m}=2)$ and self- $k$-al $(\mathrm{m}=\mathrm{k})$ functions in $k$-valued logic at $\mathrm{k} \leq 3$. And we give numerical results for clones of self-dual and self-3-al functions in 3 -valued logic. In particular, the inclusion graphs of clones of self-dual and of self-3-al functions are not a lattice.
\end{abstract}

Keywords: Discreate Mathematics, K-Valued Function Algebra, Selfdual Functions

\section{Introduction}

Multi-valued logic attracts intensive attention because of the connection with computer technology. But study of the logic is very difficult since connections of clones are very tangled and sometimes a graph of inclusions of clones is not a lattice. Some classification of all clones was given in [1].

More essential results are got for 3-valued logic. In particular, all maximal clones were found by S. V. Yablonskiy [2], all sub-maximal clones were found by D. Lau [3], all minimal clones were found by $\mathrm{B}$. Csàhàny [4], all subclones of the linear clone were found by D. Lau [3], some self-3-al clones were found by S. S. Marchenkov, J. Demetrovics, and L. Hannak [5]. More complete set of self-3-al clones is given in the article. The set of monotone clones was investigated by Machida [6]. The other types of clones were investigated very little.

The article consists of 2 sections.

Section 1 contains theoretical results. We select classes of functions from clones of functions since the classes are disjoint but clones are not disjoint. Some theorems are true only for the classes. We define conditions satisfied by functions and find theirs properties. The conditions are an extension of Post's conditions and they are used to prove some important theorems. We introduce an $m$-al operator which lives any function unchanged after using the operator $m$ times. Then we give some properties of dual and $k$-al operators. We find the numbers of relations preserved by dual and $k$-al functions.

Section 2 contains numerical results. We build the inclusion graphs of clones of self-dual and 3 -al functions and find properties of the functions.

We use the next notation. The letter $k$ is used for values of logics only. The letter $n$ is used for numbers of variables of functions only. The letters $i$ and $j$ are used as any or all values of subscripts. The letter $b$ is used as a value of variables. The letter $l$ is used as values of functions. The sign + is used as addition by modulo $k$. We use \{\} for empty sets. And we use $\bar{b}$ for any value which does not equal $b$.

We say that a relation generates a set of functions if the functions preserve the relation. We use Boolean projections of functions if values of functions are 0 or 1 whenever values of variables are 0 or 1 . Any Boolean projection is a Boolean function and can be presented by disjunctive normal form (DNF). We use sign ";" to separate values of variables from values of functions in lines of tables of functions. We call a sequence of values of variables a tuple and we can present a line of a table of a function as a tuple, , where the tuple contains values of variables and $l$ is a value of the function. We call the values of variables components of the tuple. 


\section{Theoretical Results in K-valued Logic}

\subsection{Classes of Compositions of Function}

Clones of functions are called sometimes classes. This is not well since classes must be disjoint. But clones are not disjoint, they can contain other clones. And a clone can belong to several clones.

Below we give a definition of disjoint classes. We use them since some theorems will true for classes only. And some properties of clones are in classes only.

Definition A class of a clone is a set of all functions to be generators of the clone.

Further we call classes of functions briefly classes. A name of a class is a name of a clone containing the class.

Theorem. Classes are disjoin.

Proof. Any function generates a clone set. Every function of a class generates its clone $X$. This function cannot belong to a class of a clone contained in $X$ since, by definition, the function does not belong to any clone contained in $X$. And the function cannot belong to a class of another clone since the function generates only the clone $X$.

Corollary. Every function belongs to one of classes.

Proof. Every function generating a clone belongs only to the class of the clone.

Hence, classes give a classification of functions.

If we remove all clones contained in any clone then we get a class of the clone. A function generating a clone is a member of one-membered basis. If a clone has no one-membered basis then we get an empty class after deleting other clones in the clone, since every function of a multi-membered basis belongs to one of deleted clones. But empty classes must be absent in any classification. Then clones with multi-valued bases are fictitious since they are useless for the classification of functions.

\subsection{Family of Functions with the Same Diagonal}

We will call families of functions with the same diagonal briefly families.

Families were introduced by Post. There are 4 families in two-valued logic: $\alpha, \beta, \gamma, \delta$. Post used the families to build his classification of Boolean functions.

Families of functions exist in all $k$-valued logics too. The number of these families is $2^{k}$. We will use them to classify some sets of clones.

The family $\alpha$ is defined standardly: $f(x, \ldots, x)=x$. The family $\delta$ is defined standardly, too: $f(x, \ldots, x) \neq x$. Definitions of the other families are special in $k$-valued logics but they are an extension of Post's definitions.

We denote all types of families by $\left\{j_{1}, \ldots, j_{m}\right\}$, where $j_{i} \in\{0, \ldots, k-1\}$ and where $0 \leq m \leq k-1$.

Definition A function $f$ belongs to a family $\left\{j_{1}, \ldots, j_{m}\right\}$ if $f(x, \ldots, x)$ is equal to $x$

at $x=j_{1} \vee \ldots \vee x=j_{m}$, and is not equal to $x$ at $x \neq j_{1} \wedge \ldots \wedge x \neq j_{m}$.
Hence, the $\alpha$ family is denoted by $\{0,1, \ldots, k-1\}$ and the $\delta$ family is denoted by \{\} . A constant $j$ belongs to the family $\{j\}$ because a diagonal of a constant is this constant. The $\beta$ family of two-valued logic is denoted by $\{1\}$ and the $\gamma$ family is denoted by $\{0\}$.

The denotation $\left\{j_{1}, \ldots, j_{m}\right\}$ means that diagonal of a function preserves values $j_{1}, \ldots, j_{m}$, i.e., if all variables of a function equal a $j_{i}$ then the value of the function equals the $j_{i}$. But the function does not preserve the other values.

And sets of functions of families with the same $m$ are isomorphic with respect to compositions.

The next theorem is very useful in $k$-valued logic.

Theorem. Any function of a family $X=\left\{j_{1}, \ldots, j_{m}\right\}$ generates functions of a family $Y \supseteq X$.

Proof. The operators of permutation and identification do not change a family of a function. The operator of substitution can change a family of a function.

Let functions $f_{1}^{n_{1}}, f_{2}^{n_{2}}$ be of the same family $X$, $f\left(x_{1}, \ldots, x_{n_{1}+n_{2}-1}\right)=f_{1}^{n_{1}}\left(f_{2}^{n_{2}}\left(x_{1}, \ldots, x_{n_{2}}\right), x_{n_{2}+1}, \ldots, x_{n_{1}+n_{2}-1}\right)$, and denotation of family of $f$ be $Y$.

We have $f(j, \ldots, j)=j$ for every $j \in X$. In this case $j \in Y$ for all $j \in X$. Then $Y \supseteq X$ because $f(j, \ldots, j)$ can equal $j$ for some $j \notin X$. But if $f(j, \ldots, j) \neq j$ for all $j \notin X$ then $Y=X$. The proof is finished. $\square$

Corollary. A function of the family $\alpha$ generates only function of this family. A function of the family $\delta$ may generate functions of any family.

Proof. The denotation of family $\alpha$ is the set $X=\{0, \ldots, k-1\}$ and there is no family with denotation $Y$ such that $Y \supset X$. The family $\delta$ has the denotation $X=\{\}$ and $Y \supseteq X$ for every family with denotation $Y$.

Families realize a natural classification of functions because they are disjoint.

\subsection{Classes and Families}

Functions of a clone can belong to several families. But functions of a class belong to one family only, as it follows from the next theorem.

Theorem. If a function of a class belongs to a family then all functions of the class belong to the family.

Proof. Let $k=3$ and let a family be $\left\{j_{1}, \ldots, j_{m}\right\}$ for $0 \leq m \leq 3$.

It is enough to use only families with $m=3$ since the proof of the other families is analogous. Families with $m=3$ are $\alpha,\{0,1,2\},\{0,1, \overline{2}\},\{0, \overline{1}, 2\},\{\overline{0}, 1,2\},\{0, \overline{1}, \overline{2}\}$, $\{\overline{0}, 1, \overline{2}\},\{\overline{0}, \overline{1}, 2\}, \delta$, where $\bar{b}$ has any value except $b$.

The operators of permutation and identification of variables do not change a family.

Then we use the operator of substitution of the first variable 
in a function $f^{n_{1}}$ by a function $f^{n_{2}}$. Both functions belong to the same family.

If $f^{n_{1}}$ and $f^{n_{2}}$ belong to $\alpha$ family then the function $f^{n_{1}}\left(f^{n_{2}}\left(x_{1}, \ldots, x_{n_{2}}\right), x_{n_{2}+1}, \ldots, x_{n_{1}+n_{2}-1}\right)$ belongs to $\alpha$ family since the function equals $x$ when all $x_{i}$ equal $x$.

If $f^{n_{1}}$ and $f^{n_{2}}$ belong to $\{0,1, \overline{2}\}$ family then $f^{n_{1}}\left(f^{n_{2}}(0, \ldots, 0), 0, \ldots, 0\right)=0 \quad, \quad f^{n_{1}}\left(f^{n_{2}}(1, \ldots, 1), 1, \ldots, 1\right)=1$, $f^{n_{1}}\left(f^{n_{2}}(2, \ldots, 2), 2, \ldots, 2\right)=b$, where $b$ has any value. If $b=2$ then we have $\alpha$ family. If $b=\overline{2}$ then we have $\{0,1, \overline{2}\}$ family. Hence, if a function of a class belongs to the family $\{0,1, \overline{2}\}$ then all functions of the class belong to the family, since $\alpha$ functions belong to another class.

We will get the same result for families $\{0, \overline{1}, 2\}$ and $\{\overline{0}, 1,2\}$.

If $f^{n_{1}}$ and $f^{n_{2}}$ belong to $\{0, \overline{1}, \overline{2}\}$ family then $f^{n_{1}}\left(f^{n_{2}}(0, \ldots, 0), 0, \ldots, 0\right)=0, \quad f^{n_{1}}\left(f^{n_{2}}(1, \ldots, 1), 1, \ldots, 1\right)=b_{1}$, $f^{n_{1}}\left(f^{n_{2}}(2, \ldots, 2), 2, \ldots, 2\right)=b_{2}$, where $b_{1}$ and $b_{2}$ have any value. If $b_{1}=1 \wedge b_{2}=2$ then we have $\alpha$ family. If $b_{1}=0 \wedge b_{2}=\overline{2}$ or $b_{1}=\overline{1} \wedge b_{2}=2$ then we have $\{0,1, \overline{2}\}$ or $\{0, \overline{1}, 2\}$ family. If $b_{1}=\overline{0} \wedge b_{2}=\overline{2}$ then we have $\{0, \overline{1}, \overline{2}\}$ family. Hence, if a function of a class belongs to the family $\{0, \overline{1}, \overline{2}\}$ then all functions of the class belong to the family, since otherwise the function belongs to another class.

We will get the same result for families $\{\overline{0}, 1, \overline{2}\}$ and $\{\overline{0}, \overline{1}, 2\}$.

Let $f^{n_{1}}$ and $f^{n_{2}}$ belong to $\delta$ family. In this case if a function of a class belongs to $\delta$ family then all functions of the class belong to the family, since otherwise a function belongs to another class.

We will get the same result fo $k>3$.

\subsection{Conditions of Functions}

Any function must satisfy some conditions.

There are 2 conditions for any function in 2-valued logic. They are $a^{\mu}$ and $A^{\mu}$ [7]. We will denote them by $a_{0}^{\mu}$ and $a_{1}^{\mu}$. But there are $k$ conditions in $k$-valued logic. They are $a_{0}^{\mu}, \ldots, a_{k-1}^{\mu}$. This holds for all $k$ including $k=\omega$.

Definition A function satisfies a condition

- $a_{l}^{\mu}(0 \leq \mu<\omega)$ if any $\mu$ tuples, have a column with value $l$ only, and if there are $\mu+1$ tuples $_{l}$ which have not a column with value $l$ only,

- $a_{l}^{\omega+m}(0 \leq m<\omega)$ if we have $m$ tuples $_{l}$ and all they have a column with value $l$.

If a function does not satisfy a condition of the first part of definition then the function satisfies a condition of the second part of definition. Indeed, let a function do not satisfy $a_{l}^{\mu}$. Then all tuples ${ }_{l}$ are absent or $m$ tuples, exist but $m+1$ tuples $_{l}$ are absent. In the former case the function satisfies the condition $a_{l}^{\omega}$. In the latter case the function satisfies the condition $a_{l}^{\omega+m}$.

By definition, if one of tuples, of a function does not contain value $l$ of variables then the function satisfies the condition $a_{l}^{0}$. And by definition, if a function takes not all values then the function satisfies the condition $a_{l}^{\omega}$ for absent $l$.

A constant $j$ satisfies conditions $a_{j}^{0}$ and $a_{i}^{\omega}$ for $i \neq j$. Any delta function $f$ satisfies $a_{i}^{0}$ for all $i$ since $f(x, \ldots, x) \neq x$.

The next lemma is very useful.

Lemma. If a function satisfies a condition $a_{l}^{\mu}$ for some $l$ at $\mu<\omega$ then there are $\mu+1$ tuples $_{l}$ and $\mu+1$ columns in them such that every column contains only one value $\bar{l}$, and every tuple ${ }_{l}$ has only one value $\bar{l}$ in the columns.

Proof. By definition, if a function satisfies a condition $a_{l}^{\mu}$ for $\mu<\omega$ then there are $\mu+1$ different tuples, not satisfying the condition. We take only columns of the tuples ${ }_{l}$ such that every of the columns has only one value equal to $\bar{l}$. Such columns exist since otherwise some $\mu$ of $\mu+1$ tuples different columns with only one value $\bar{l}$ is less than $\mu+1$

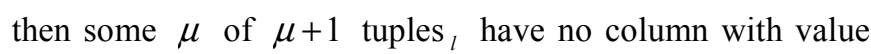
$l$ only. But every $\mu$ of $\mu+1$ tuples, have a column with value $l$ only, iff there are $\mu+1$ different columns with only one value $\bar{l}$. Every tuple, has only one value $\bar{l}$ in the columns since the columns are different.

If $\mu<\omega$ then we can take lines and columns of any table such that values not equaling $l$ form a diagonal:

$$
\begin{array}{ccccc}
b_{1} & l & l & \ldots & l \\
l & b_{2} & l & \ldots & l \\
l & l & b_{3} & \ldots & l \\
. & . & . & . & . \\
l & l & l & \ldots & b_{\mu+1}
\end{array}
$$

where any $b_{i}$ does not equal $l$.

We will call the subset of tuples sub-tuples $_{l}$.

\subsection{Classes and Conditions}

Functions of a clone can satisfy several conditions. But functions of a class satisfy one condition only, as it follows 
from the next theorem. For simplification of the proof we say that a function has $\mu$ for some $l$, if it satisfies a condition $a_{l}^{\mu}$.

Theorem. If a function of a class satisfies a condition $a_{l}^{\mu_{0}}$ at $0 \leq \mu_{0}<\omega$ for some $l$ then all functions of the class satisfy the condition.

Proof. Let a function $f_{0}^{n_{0}}$ belong to a class and satisfy a condition $a_{l}^{\mu_{0}}$ at $0 \leq \mu_{0}<\omega$ for some $l$. We must prove that $f_{0}^{n_{0}}$ generates the class of functions $f$ with $\mu=\mu_{0}$. For that we use Mal'cev's operators of compositions [8].

The operator of permutation of variables does not change the condition since a permutation of variables is the permutation of columns, but the permutation does not change values into columns.

The operator of identification of variables does not change the conditions, too. Indeed, we can have the next $\mu_{0}+1$ sub-tuples, of tuples, of the function $f_{0}^{n_{0}}$ :

$\begin{array}{ccccc}b_{1} & l & l & \ldots & l \\ l & b_{2} & l & \ldots & l \\ l & l & b_{3} & \ldots & l \\ \cdot & \cdot & \cdot & . & \cdot \\ l & l & l & \ldots & b_{\mu_{0}+1}\end{array}$

where any $b_{i}$ has any value except $l$. If we identify some variables then a part of the sub-tuples, can be deleted. If no sub-tuple $_{l}$ is deleted then $\mu=\mu_{0}$, where $\mu$ belongs to the $f$ and $\mu_{0}$ belongs to the $f_{0}^{n_{0}}$. Otherwise we have one or more columns with value $l$ only. Then $\mu>\omega$ but functions with the $\mu$ belong to another classes since functions with the $\mu$ cannot generate functions with $\mu<\omega$ (functions of a class must generate one to other). So the theorem holds in this case.

Now we must prove that the operator of substitution of the first variable in a function $f_{1}^{n_{1}}$ with $\mu_{0}$ by the function $f_{2}^{n_{2}}$ does not change the condition. The operator gives

$$
f^{n_{1}+n_{2}-1}\left(x_{1}, \ldots, x_{n_{1}+n_{1}-1}\right)=f_{1}^{n_{1}}\left(f_{2}^{n_{2}}\left(x_{1}, \ldots, x_{n_{2}}\right), x_{n_{2}+1}, \ldots, x_{n_{1}+n_{2}-1}\right)
$$

We must prove that the function $f^{n_{1}+n_{2}-1}$ has $\mu=\mu_{0}$. For that we will use tuples, of $f^{n_{1}+n_{2}-1}$. If we replace $f_{2}^{n_{2}}\left(x_{1}, \ldots, x_{n_{2}}\right)$ in the tuples, by values of $f^{n_{2}}$ then we have the next sub-tuples ${ }_{l}$ of $f^{n_{1}+n_{2}-1}$ :

$\begin{array}{cccccc}b_{1} & l & l & \ldots & l & l \\ l & b_{2} & l & \ldots & l & l \\ \cdot & \cdot & . & . & \cdot & \cdot \\ l & l & l & \ldots \ldots & l & b_{\mu+1}\end{array}$

where any $b_{i}$ has any value except $l$.

It cannot be $\mu<\mu_{0}$ since every $\mu_{0}$ tuples $_{l}$ must have a column with values $l$ only, then the $\mu$ tuples, have a column with values $l$ only.

Hence, $\mu \geq \mu_{0}$. In reality, $\mu=\mu_{0}$. Indeed, let functions $f_{1}$ with $\mu_{1}$ and $f_{2}$ with $\mu_{2}$ belong to the same class. We have $\mu_{2} \geq \mu_{1}$ since $f_{1}$ generates $f_{2}$. And we have $\mu_{1} \geq \mu_{2}$ since $f_{2}$ generates $f_{1}$. Hence, $\mu_{1}=\mu_{2}$. $\square$

Corollary. If a function of a class satisfies a condition $a_{l}^{\mu_{0}}$ at $0 \leq \mu_{0}<\omega$ for some $l$ then all functions of the family in the clone of the class satisfy the condition with $\mu \geq \mu_{0}$ for the $l$.

Proof. By previous proof a function with $\mu_{0}$ generates functions with $\mu \geq \mu_{0}$.

Hence, if functions satisfy different $a_{l}^{\mu}$ with $\mu<\omega$ then the functions generate different clones. We will use the property below.

\subsection{Boolean Projections}

Boolean projections are used at classification of clones.

Definition A function $f$ has a Boolean projection if $f\left(x_{1}, \ldots, x_{n}\right) \in\{0,1\}$ when all $x_{i} \in\{0,1\}$. We denote a Boolean projection by prf. A clone of functions has a Boolean projection if every function of the clone has some Boolean projection.

Lemma. If a function has a Boolean projection then a clone generated by the function has a Boolean projection.

Proof. It is enough to prove that a Boolean projection of a function and and a Boolean projection of any composition of the function belong to the same clone.

If we permute or identify variables of a function then we permute or identify variables in the Boolean projection of the function. Then the initial Boolean projection and the new Boolean projection belong to the same clone.

Let functions $f^{n_{1}}, f^{n_{2}}$ belong to the same clone and have Boolean projections. The operation of substitution gives the function that $f^{n_{1}+n_{2}-1}$ equals $f^{n_{1}}\left(f^{n_{2}}\left(x_{1}, \ldots, x_{n_{2}}\right), x_{n_{2}+1}, \ldots, x_{n_{1}+n_{2}-1}\right)$. The Boolean projection of the function is a result of substitution of the Boolean projections. Indeed, $f^{n_{2}} \in\{0,1\}$ if its $x_{i} \in\{0,1\}$. And $f^{n_{1}+n_{2}-1} \in\{0,1\}$ if $x_{1}, \ldots, x_{n_{1}+n_{2}-1}$ are members of $\{0,1\}$. Hence, the new Boolean projection and the initial Boolean projection belong to the same clone. $\square$

The set of Boolean functions is countable. We can suppose 
that any set of clones is not continual if these clones have Boolean projections and $k>2$. But this is not proved now.

\subsection{Dual and M-Al Operators}

The operators are used to build clones of functions.

Definition A unary operator $O$ over functions is $\mathrm{m}$-al if using the operator $m$ times, we leave any function unchanged. We call a function $f_{1} \mathrm{~m}$-al to $f_{2}$, if $f_{1}=O f_{2}$ and $O$ is $m$-al. We call a set $X_{1}$ of functions m-al to a set $X_{2}$ of functions if every function of $X_{1}$ is $m$-al to a function of $X_{2}$.

Dual operators are particular cases of $m$-al operators. But now one calls any $m$-al operator "dual". It is not well since $m$-al operators with different $m$ have very different properties.

Some operators can be functions. For example, the identity function $f(x)=x$ is 1 -al operator and the negation (in Post logic) $f(x)=x+1$ is $k$-al operator. Here values of $x$ are functions. We denote the operators by $s_{i}^{m}(x)$, where $i$ means an ordinal number of $m$-al operator.

Operators $s_{i}^{m}$ are not used since they do not preserve compositions of functions.

Indeed, we say that an operator $O$ preserves compositions of functions if a function $f$ is $m$-al to a function $g$ and the clone generated by $f$ is $m$-al to the clone generated by $g$.

The next lemma states that operators $s_{i}^{m}$ do not preserve compositions of functions.

Lemma. Let a function $f$ be $m$-al to a function $g$ Then it can be that the clone generated by $f$ is not $m$-al to the clone generated by $g$.

Proof. Compositions of functions use operators of permutation and identification of variables in functions and the operator of substitution of the first variable into a function by a function. It is enough to use only the last operator for the proof.

Let functions $f_{1}$ and $f_{2}$ be $m$-al to functions $g_{1}$ and $g_{2}$ with respect to an operator $s_{i}^{m}$ :

$$
f_{1}\left(x_{1}, \ldots, x_{n_{1}}\right)=s_{i}^{m}\left(g_{1}\left(x_{1}, \ldots, x_{n_{1}}\right)\right), \quad f_{2}\left(x_{1}, \ldots, x_{n_{2}}\right)=s_{i}^{m}\left(g_{2}\left(x_{1}, \ldots, x_{n_{2}}\right)\right)
$$

and let functions $f_{0}$ and $g_{0}$ be

$$
\begin{gathered}
f_{0}\left(x_{1}, \ldots, x_{n_{1}+n_{2}-1}\right)=f_{1}\left(f_{2}\left(x_{1}, \ldots, x_{n_{2}}\right), x_{n_{2}+1}, \ldots, x_{n_{1}+n_{2}-1}\right) \\
g_{0}\left(x_{1}, \ldots, x_{n_{1}+n_{2}-1}\right)=g_{1}\left(g_{2}\left(x_{1}, \ldots, x_{n_{2}}\right), x_{n_{2}+1}, \ldots, x_{n_{1}+n_{2}-1}\right)
\end{gathered}
$$

We shall prove that it can be

$$
f_{0}\left(x_{1}, \ldots, x_{n_{1}+n_{2}-1}\right) \neq s_{i}^{m}\left(g_{0}\left(x_{1}, \ldots, x_{n_{1}+n_{2}-1}\right)\right)
$$

Using (1) and (2), we have

$$
\left.f_{0}\left(x_{1}, \ldots, x_{n_{1}+n_{2}-1}\right)=s_{i}^{m}\left(g_{1}\left(f_{2}\left(x_{1}, \ldots, x_{n_{2}}\right)\right), x_{n_{2}+1}, \ldots, x_{n_{1}+n_{2}-1}\right)\right)
$$

Using (3), we have

$$
s_{i}^{m}\left(g_{0}\left(x_{1}, \ldots, x_{n_{1}+n_{2}-1}\right)\right)=s_{i}^{m}\left(g_{1}\left(g_{2}\left(x_{1}, \ldots, x_{n_{2}}\right), x_{n_{2}+1}, \ldots, x_{n_{1}+n_{2}-1}\right)\right)
$$

Comparing (5) and (6), we see that if $f_{2} \neq g_{2}$ then (4) holds.

Some operators are not functions and can preserve compositions of functions. This property of the operators is named the duality (more correctly, $m$-ality) principle. These operators are not $s_{i}^{m}$ but they use $s_{i}^{m}$ (below we take $s_{i}$ instead of $s_{i}^{m}$ and we use superscripts as powers, for example, $\left.s_{i}^{-1}=s_{i}^{k-1}\right)$ :

$$
O f\left(x_{1}, \ldots, x_{n}\right)=s_{i}^{-1}\left(f\left(s_{i}\left(x_{1}\right), \ldots, s_{i}\left(x_{n}\right)\right)\right)
$$

Further we use only these operators to create clones since only they preserve compositions.

The functions

$$
s_{i}=\left(\begin{array}{cccc}
0 & 1 & \ldots & k-1 \\
b_{1} & b_{2} & \ldots & b_{k-1}
\end{array}\right)
$$

are used as two-ary relations

$$
\rho_{i}=\left\{\begin{array}{cccc}
0 & 1 & \ldots & k-1 \\
b_{1} & b_{2} & \ldots & b_{k-1}
\end{array}\right\},
$$

and the relations generate clones, i.e., every $s_{i}$ has corresponding clone. We will say conditionally that $\rho_{i}=s_{i}$.

There are $m$ clones, one $X_{1}$ of them is $m$-al to an $X_{2}$, where $X_{2}$ is $m$-al to an $X_{3}$, and so on. Some clones can be equal. And there are $m$ sets of the clones. All of the sets are isomorphic with respect to inclusions of clones. This means that we can use only one of the sets to build all inclusion diagrams of their clones. The dual and the $k$-al functions are presented more interest.

\subsection{Dual Operators}

A dual operator can be a function. Let the function be $s(x)$. There are 3 functions in 3-valued logic:

$$
s_{1}=\left(\begin{array}{lll}
0 & 1 & 2 \\
0 & 2 & 1
\end{array}\right), \quad s_{2}=\left(\begin{array}{lll}
0 & 1 & 2 \\
1 & 0 & 2
\end{array}\right), \quad s_{3}=\left(\begin{array}{lll}
0 & 1 & 2 \\
2 & 1 & 0
\end{array}\right) .
$$

where $s_{1}$ is presented by cycles $(0)(1,2), s_{2}$ is presented by cycles $(0,1)(2)$, and $s_{3}$ is presented by cycles $(0,2)(1)$.

There are 9 functions in 4-valued logic:

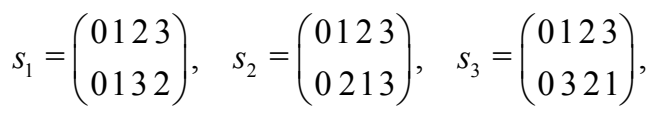




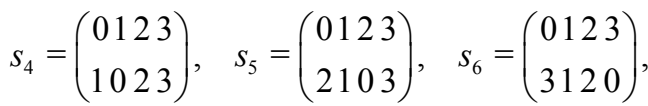

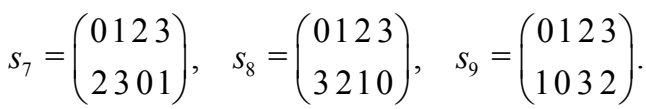

where all $s_{i}$ are presented by cycles of lengths 1 and 2 or of length 2 only. And relations generating clones are $\rho_{i}=s_{i}$. Clones generated by $\rho_{1}-\rho_{6}$ are isomorphic with respect to compositions. And clones generated by $\rho_{7}-\rho_{9}$ are isomorphic too.

The next characteristics of the operators exist for all $k$ :

○ $s_{i}^{-1}(x)=s_{i}(x)$,

$\circ$ relations $\rho_{i}$ generating clones equal $s_{i}$,

- the functions $s_{i}(x)$ are presented by cycles of lengths 1 and 2 or of length 2 only, and inverse is true: 1-ary functions with the cycles are $s_{i}(x)$,

$\circ$ if some $s_{i}$ have cycles only of length 2 then the number of the $s_{i}$ is $1 * 3 * 5 * \ldots *(k-1)$ and then clones generated by their $\rho_{i}$ are maximal, but clones generated by the other $\rho_{i}$ are not maximal ([]),

- different $\rho_{i}$ generate different clones, i.e., $\rho_{i}$ generating the same clones are absent,

- if some $s_{i}$ have the same number of cycles and the cycles have the same lengths, then their $\rho_{i}$ generate isomorphic clones,

$$
\begin{aligned}
& \left(\begin{array}{l}
k \\
2
\end{array}\right)+\left(\begin{array}{c}
k \\
2
\end{array}\right)\left(\begin{array}{c}
k-2 \\
2
\end{array}\right) / 2 !+\left(\begin{array}{c}
k \\
2
\end{array}\right)\left(\begin{array}{c}
k-2 \\
2
\end{array}\right)\left(\begin{array}{c}
k-4 \\
2
\end{array}\right) / 3 !+\ldots, \text { where } \\
& \left(\begin{array}{l}
m_{1} \\
m_{2}
\end{array}\right)=0 \text { if } m_{1}<m_{2} .
\end{aligned}
$$

The next theorem proves the last property.

Theorem. The number of functions $s_{i}(x)$ in $k$-valued logic is

$$
\left(\begin{array}{l}
k \\
2
\end{array}\right)+\left(\begin{array}{l}
k \\
2
\end{array}\right)\left(\begin{array}{c}
k-2 \\
2
\end{array}\right) / 2 !+\left(\begin{array}{l}
k \\
2
\end{array}\right)\left(\begin{array}{c}
k-2 \\
2
\end{array}\right)\left(\begin{array}{c}
k-4 \\
2
\end{array}\right) / 3 !+\ldots
$$

Proof. The number of 1-ary functions with one cycle $\left(i_{1}, i_{2}\right)$ of length 2 (all other cycles have length 1 ) is $\left(\begin{array}{l}k \\ 2\end{array}\right)$. The number of 1-ary functions with two cycles $\left(i_{1}, i_{2}\right)\left(i_{3}, i_{4}\right)$ $\left(i_{1}, i_{2} \neq i_{3}, i_{4}\right)$ is $\left(\begin{array}{l}k \\ 2\end{array}\right)\left(\begin{array}{c}k-2 \\ 2\end{array}\right)$. But cycles $\left(i_{1}, i_{2}\right)\left(i_{3}, i_{4}\right)$ and $\left(i_{3}, i_{4}\right)\left(i_{1}, i_{2}\right)$ are the same. The number of 1-ary functions with 3 cycles $\left(i_{1}, i_{2}\right)\left(i_{3}, i_{4}\right)\left(i_{5}, i_{6}\right)$ is $\left(\begin{array}{l}k \\ 2\end{array}\right)\left(\begin{array}{c}k-2 \\ 2\end{array}\right)\left(\begin{array}{c}k-4 \\ 2\end{array}\right)$. But there are 3 ! permutations of the pairs. And so on.

\subsection{Self-Dual Functions}

We will call clones of self-dual (auto-dual) functions briefly clones. We will prove that the number of clones is infinite if $k \geq 3$. But this number is finite if $k=2$ [7].

Definition We call a function self-dual with respect to a dual operator if the function is dual to itself, i.e., it remains unchanged after one application of the dual operator.

A clone of self-dual functions can be presented as $\operatorname{Pol}\left(\rho_{i}\right)$, where $\rho_{i}=s_{i}$. So the number of clones generated by the relations equals the number of $s_{i}(x)$.

The other property is given by the next theorem.

Theorem. Clones of self-dual functions satisfy conditions $a_{l}^{\mu}$ at $k \geq 3$ for any finite $\mu$ and for any $l$ not presented in cycles of length 1 .

Proof. It is enough to prove the theorem in 3-valued logic because in $k$-valued logic the proof is analogous.

There are 3 dual operators and $3 \operatorname{Pol}\left(\rho_{i}\right)$. Corresponding clones are isomorphic and we use only the relation

\begin{tabular}{|c|c|c|c|c|c|}
\hline$x_{1}$ & $x_{2}$ & $x_{3}$ & $\ldots$ & $\boldsymbol{x}_{n}$ & $f$ \\
\hline 2 & 1 & 1 & $\ldots$ & 1 & 1 \\
\hline 1 & 2 & 1 & $\ldots$ & 1 & 1 \\
\hline 1 & 1 & 2 & $\ldots$ & 1 & 1 \\
\hline . & . & . & . & . & . \\
\hline 1 & 1 & 1 & $\ldots$ & 2 & 1 \\
\hline 1 & 2 & 2 & $\ldots$ & 2 & 2 \\
\hline 2 & 1 & 2 & $\ldots$ & 2 & 2 \\
\hline 2 & 2 & 1 & $\ldots$ & 2 & 2 \\
\hline 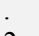 & . & . & . & . & . \\
\hline 2 & 2 & 2 & $\ldots$ & 1 & 2 \\
\hline
\end{tabular}
$\rho=\left\{\begin{array}{lll}0 & 1 & 2 \\ 0 & 2 & 1\end{array}\right\}$. The relation equals $s_{1}$.

Let $\mathrm{f}\left(x_{1}, \ldots, \mathrm{x}_{\mathrm{n}}\right)$ be functions of $n \geq 3$. A function of some $n$ is presented in table 1 .

Table 1. Values of function $f\left(x_{1}, \ldots, x_{n}\right)$. Absent values are 0.

We must prove the next characteristics:

- the functions preserve the relation $\rho=\left\{\begin{array}{lll}0 & 1 & 2 \\ 0 & 2 & 1\end{array}\right\}$.

- the functions satisfy the conditions $\left\{a_{0}^{0}, a_{1}^{n-1}, a_{2}^{n-1}\right\}$,

- the functions of different $n$ generate different clones.

Indeed, the function preserves $\rho$ because

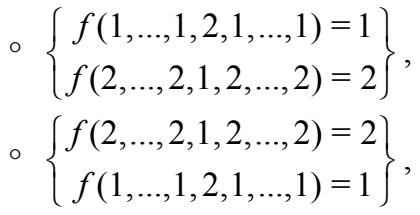

- the other pairs of lines contain the pair of function's values $\left\{\begin{array}{l}0 \\ 0\end{array}\right\}$ or they are absent because they contain the pair $\left\{\begin{array}{l}1 \\ 1\end{array}\right\}$ or the pair $\left\{\begin{array}{l}2 \\ 2\end{array}\right\}$ of values of variables.

The functions satisfy the condition $a_{0}^{0}$ because $f(1, \ldots, 1)=0$. They satisfy conditions $a_{1}^{n-1}$ and $a_{2}^{n-1}$ 
because every $n-1$ lines have a column with values 1 whenever $f=1$ and every $n-1$ lines have a column with values 2 whenever $f=2$.

By theorem in 2.5, the function generates different clones for different $n$.

Hence, the number of the clones is countable. $\square$

\subsection{On K-al Operators}

There are 2 functions to be $k$-al operators in 3 -valued logic:

$$
s_{1}=\left(\begin{array}{lll}
0 & 1 & 2 \\
1 & 2 & 0
\end{array}\right), \quad s_{2}=\left(\begin{array}{lll}
0 & 1 & 2 \\
2 & 0 & 1
\end{array}\right)
$$

Relations $\rho_{1}=s_{1}$ and $\rho_{2}=s_{2}$ generate the same clones.

There are 6 functions to be $k$-al operators in 4 -valued logic:

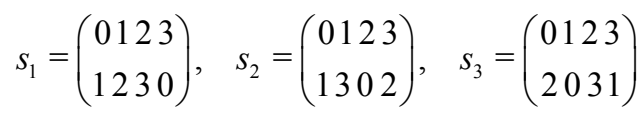

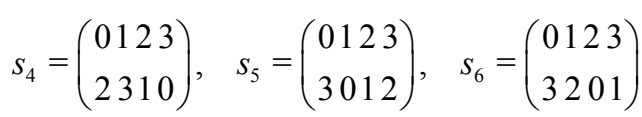

Every pair of relations $\left(\rho_{1}=s_{1}, \rho_{5}=s_{5}\right)$, $\left(\rho_{2}=s_{2}, \rho_{3}=s_{3}\right)$, or $\left(\rho_{4}=s_{4}, \rho_{6}=s_{6}\right)$ generates the same clones. Clones generated by these pairs are isomorphic with respect to compositions.

All operators $s_{i}$ of any $k$-valued logic have the next characteristics:

- $s_{i}^{-1}(x)=s_{i}^{k-1}(x)$

$\circ$ relations $\rho_{i}$ generating clones equal $s_{i}$,

- clones generated by any $\rho_{i}$ are not maximal,

- if some $s_{i}$ have the same number of cycles, the cycles have the same lengths, and the $\rho_{i}$ generate different clones then the $\rho_{i}$ generate isomorphic clones,

○ if $k=2^{m}$ then $k / 2$ of $\rho_{i}$ generate the same clones;

$\circ$ if $k=p^{m} \wedge p \geq 3$, where $p$ is prime number and $m \geq 1$, then all $\rho_{i}$ generate the same clones,

○ if $k=p_{1}^{m_{1}} * \ldots p_{j}^{m_{j}} \wedge j \geq 2$ then all $\rho_{i}$ generate different clones,

- there are $(k-1)$ ! functions $s_{i}$ with one cycle,

$\circ$ if $k=p^{m}$ then every $s_{i}$ has one cycle,

$\circ$ if $k=p_{1}^{m_{1}} \ldots p_{l}^{m_{l}}$ then we have $s_{i}$ with one cycle and $s_{i}$ with several cycles such that $l_{j}$ of the cycles have length $p_{j}^{m_{j}}$ and $l_{0}$ have length 1 , this is true for all $l_{j}$ and $l_{0}$ which defined by $l_{0}+l_{1} * p_{1}^{m_{1}}+\ldots+l_{l} * p_{l}^{m_{l}}=k$.

We shall prove three last properties.

Theorem. There are $(k-1)$ ! functions $s_{i}$ with one cycle.
Proof. Let $s_{i}$ have one cycle. Then

$$
s_{i}=\left(\begin{array}{ccccc}
0 & b_{1} & \ldots & b_{k-2} & b_{k-1} \\
b_{1} & b_{2} & \ldots & b_{k-1} & 0
\end{array}\right)
$$

where the first row is a sequence $\left(0, b_{1}, b_{2}, \ldots, b_{k-1}\right)$, all $b_{i}$ are mutually unequal, and all $b_{i}$ do not equal 0 . Then $b_{1}$ can have $k-1$ different values, $b_{2}$ can have $k-2$ different values,..., $b_{k-1}$ has only one value. Hence, there are $(k-1)$ ! different values and $(k-1)$ ! different $s_{i}$. $\square$

Theorem If $k=p^{m}$ then every $s_{i}$ has one cycle.

Proof by contradiction. We must prove that $s_{i}^{m}=s_{i}$ only if $m \neq k$.

Let $s_{i}$ have 2 cycles of lengths $m_{1}$ and $m_{2}\left(m_{1}+m_{2} \leq k\right)$ and let the other cycles have length 1 :

$$
s_{i}=\left(\begin{array}{cccccccccc}
0 & b_{1} & \ldots & b_{m_{1}-2} & b_{m_{1}-1} & b_{m_{1}} & \ldots & b_{m_{1}+m_{2}-2} & b_{m_{1}+m_{2}-1} & \ldots \\
b_{1} & b_{2} & \ldots & b_{m_{1}-1} & 0 & b_{m_{1}}+1 & \ldots & b_{m_{1}+m_{2}-1} & b_{m_{1}} & \ldots
\end{array}\right)
$$

The first $m_{1}$ components become without change after $s_{i}^{m_{1}}$. And the next $m_{2}$ component become without change after $s_{i}^{m_{2}}$. If $m_{1}=m_{2}$ then $s_{i}=s_{i}^{m_{1}}$ but $m_{1}<k$.

If $m_{1}$ and $m_{2}$ are relatively prime then $s_{i} \neq s_{i}^{m_{1}} \wedge s_{i} \neq s_{i}^{m_{2}}$ but $s_{i}=s_{i}^{m_{1}{ }^{*} m_{2}}$ and $k \neq m_{1} * m_{2}$.

If $m_{1} \neq m_{2}$ and $m_{1}$ and $m_{2}$ are not relatively prime then $s_{i}=s_{i}{ }^{m_{1}{ }^{*} m_{2} / m_{0}^{2}}$ where $m_{0}$ is the common deviser of $m_{1}$ and $m_{2}$. But $m_{1} * m_{2} / m_{0}^{2} \neq k$

We will get the same result if $s_{i}$ has 3 or more cycles.

Theorem. If $k=p_{1}^{m_{1}} \ldots p_{l}^{m_{l}}$ then we have $s_{i}$ with one cycle and $s_{i}$ with several cycles such that $l_{j}$ of the cycles have length $p_{j}^{m_{j}}$ and $l_{0}$ have length 1 , this is true for all $l_{j}$ and $l_{0}$ which defined by $l_{0}+l_{1} * p_{1}^{m_{1}}+\ldots+l_{l} * p_{l}^{m_{l}}=k$.

Proof. If $s_{i}=\left(\begin{array}{ccccc}0 & b_{1} & \ldots & b_{k-2} & b_{k-1} \\ b_{1} & b_{2} & \ldots & b_{k-1} & 0\end{array}\right)$ then $s_{i}^{k}=s$, i.e., the $s_{i}$ have one cycle of length $k$.

We have $s_{i} \neq s_{i}^{p_{j}^{m_{j}}}$ for $1 \leq j \leq l$ but $s_{i}=s_{i}^{p_{1}^{m_{1}} \ldots *^{*} p_{l} m_{l}}$. Hence, the $s_{i}$ have $l_{j}$ cycles of length $p_{j}{ }^{{ }_{j}}$ for all $l_{j}$ and $l_{0}$ defined by $l_{0}+l_{1} * p_{1}^{m_{1}}+\ldots+l_{l} * p_{l}^{m_{l}}=k$.

For example, if $k=2 * 3$ then we have



where $s_{1}-S_{120}$ have one cycle of length $6, s_{121}$ and all 
other $s_{i}$ have 3 cycles of lengths $1,2,3$.

If $k=2^{2} * 3$ then we have $s_{i}$ with: (1) one cycle of length $k$; (2) 7 cycles such that one of them has length 4 , one has length 3 , and 5 have length 1 ; (3) 4 cycles such that two of them have length 4 , one has length 3 , and one has length 1 ; (4) 5 cycles such that one of them has length 4 , two have length 3 and two have length 1 .

\subsection{Self-K-Al Functions}

The number of clones of self- $k$-al functions is finite at $k=2(k$-al and dual operators are the same if $k=2)$. But the number of the clones is not finite at $k>2$.

Definition We call a function $\mathrm{k}$-al to a function if the former function is obtained from the latter functions by using some $k$-al operator $k-1$ times. We call a function self-k-al if the function equals its $k$-al function.

So a function $f$ is self- $k$-al if $f\left(x_{1}, \ldots, x_{n}\right)=s_{i}^{-1}\left(f\left(s_{i}\left(x_{1}\right), \ldots, s_{i}\left(x_{n}\right)\right)\right)$.

A clone of self- $k$-al functions can be presented as $\operatorname{Pol}\left(\rho_{i}\right)$, where $\rho_{i}=s_{i}$.

The number of clones of self- $k$-al functions can be finite. We shall prove this but before we shall prove the next lemmas.

Lemma 1. If $s_{i}$ has one cycle then the numbers of tuples, of any self- $k$-al function preserving $\rho_{i}=s_{i}$ are the same for all $l$.

Proof. Let a self- $k$-al function $f$ preserve the relation

$$
\left\{\begin{array}{ccccc}
0 & b_{1} & \ldots & b_{k-2} & b_{k-1} \\
b_{1} & b_{2} & \ldots & b_{k-1} & 0
\end{array}\right\}
$$

Then every tuple ${ }_{0}$ has corresponding tuple ${ }_{b_{1}}$. Hence, the number of tuples ${ }_{0}$ equals the number of tuples ${ }_{b_{1}}$. Further, every tuple $e_{b_{1}}$ has corresponding tuple ${ }_{b_{2}}$. Hence, the number of tuples $_{b_{1}}$ equals the number of tuples ${ }_{b_{2}}$. And so on.

Corollary. If $s_{i}$ has one cycle then self- $k$-al functions generated by $\rho_{i}=s_{i}$ have all values.

Proof. If tuples ${ }_{l}$ of a function are absent for some $l$ then the function has not the value 1. By the lemma, all values of the function are absent. But such the function does not exist.

Lemma 2. Let $s_{i}$ have one cycle and let $f$ be a self- $k$-al function preserving $\rho_{i}=s_{i}$. Then values of $f\left(0, x_{2}, \ldots, x_{n}\right)$ are arbitrary but values of $f\left(\overline{0}, x_{2}, \ldots, x_{n}\right)$ depend on values of $f\left(0, x_{2}, \ldots, x_{n}\right)$.

Proof. Let

$$
\rho=\left\{\begin{array}{ccccc}
0 & b_{1} & \ldots & b_{k-2} & b_{k-1} \\
b_{1} & b_{2} & \ldots & b_{k-1} & 0
\end{array}\right\}
$$

then

$$
\begin{gathered}
f\left(b_{1}, b_{j_{1}}, b_{j_{2}}, \ldots, b_{j_{k-1}}\right)=f\left(0, b_{j_{1}-1}, b_{j_{2}-1}, \ldots, b_{j_{k-1}-1}\right) \\
f\left(b_{2}, b_{j_{1}}, b_{j_{2}}, \ldots, b_{j_{k-1}}\right)=f\left(0, b_{j_{1}-2}, b_{j_{2}-2}, \ldots, b_{j_{k-1}-2}\right)
\end{gathered}
$$

and so on. $\square$

Lemma 3. If $s_{i}$ has one cycle then self- $k$-al functions preserving $\rho_{i}=s_{i}$ belong only to $\alpha$ and $\delta$ families.

Proof. Let

$$
\rho_{i}=\left(\begin{array}{cccccc}
0 & b_{1} & b_{2} & \ldots & b_{k-2} & b_{k-1} \\
b_{1} & b_{2} & b_{3} & \ldots & b_{k-1} & 0
\end{array}\right)
$$

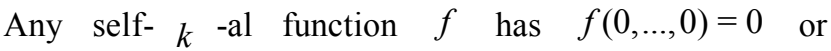
$f(0, \ldots, 0)=\overline{0}$.

If $f(0, \ldots, 0)=0$ then the function is $\alpha$. Indeed, by the first column in $\rho_{i}, f(0, \ldots, 0)=0$ implies $f\left(b_{1}, \ldots, b_{1}\right)=b_{1}$. By the second column in $\rho_{i}, f\left(b_{1}, \ldots, b_{1}\right)=b_{1}$ implies $f\left(b_{2}, \ldots, b_{2}\right)=b_{2}$. And so on. But every $b_{i}$ runs through all values.

If $f(0, \ldots, 0)=\overline{0}$ then the function is $\delta$ since $f(0, \ldots, 0)=\overline{0}$ implies $f\left(b_{1}, \ldots, b_{1}\right)=\bar{b}_{1}, \quad f\left(b_{1}, \ldots, b_{1}\right)=\bar{b}_{1}$ implies $f\left(b_{2}, \ldots, b_{2}\right)=\bar{b}_{2}$, and so on.

The same families of functions are generated by compositions.

Indeed, the operations of permutations and identification of variables do not change families. The operation of substitution does not change $\alpha$ families. But the operation can change $\delta$ families.

Let $f$ be a $\delta$ function. Then $f(f(0, \ldots, 0), 0, \ldots, 0)=0$ or $f(f(0, \ldots, 0), 0, \ldots, 0)=\overline{0}$. If $f(f(0, \ldots, 0), 0, \ldots, 0)=0$ then the function is $\alpha$ else the functions is $\delta$. $\square$

Theorem. If $s_{i}$ has one cycle then all self- $k$-al functions generated by $\rho_{i}=s_{i}$ satisfy the same conditions for all $l$. These conditions are $a_{l}^{0}, a_{l}^{1}$, or $a_{l}^{\omega+k^{n-1}}$.

Proof. The proof consists of 2 parts.

- We will prove that all self- $k$-al functions satisfy the same conditions for all $l$.

Let a self- $k$-al function preserve a relation $\left\{\begin{array}{ccccc}0 & 1 & \ldots & k-2 & k-1 \\ 1 & 2 & \ldots & k-1 & 0\end{array}\right\}$ and let the function satisfy a condition $a_{l}^{\mu}$ for some $l$.

Then we have the next sub-tuples, after permuting columns:

$$
\begin{array}{ccccc}
b_{1} & l & l & \ldots & l \\
l & b_{2} & l & \ldots & l \\
l & l & b_{3} & \ldots & l \\
\cdot & . & . & . & \cdot \\
l & l & l & \ldots & b_{\mu+1}
\end{array}
$$


where $b_{i}$ does not equal $l$.

Using the relation, we have

$$
\begin{array}{ccccc}
b_{1}+1 & l+1 & l+1 & \ldots & l+1 \\
l+1 & b_{2}+1 & l+1 & \ldots & l+1 \\
l+1 & l+1 & b_{3}+1 & \ldots & l+1 \\
. & . & \cdot & . & \cdot \\
l+1 & l+1 & l+1 & \ldots & b_{\mu+1}+1
\end{array}
$$

where $b_{i}+1$ does not equal $l+1$.

Hence, the function satisfies the same condition for $l+1$ and for other $l$ too.

We have the same result if we use the other relations for self-k-al functions, because every tuple $l_{1}$ has corresponding tuple $_{l_{2}}$.

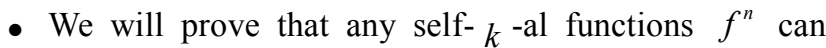
satisfy only conditions $a_{l}^{\mu}$ with $\mu=0$, with $\mu=1$, or with $\mu=\omega+k^{n-1}$. If all tuples ${ }_{0}$ have a column with values 0 only then $\mu=\omega+k^{n-1}$ since the number of the tuples ${ }_{0}$ is $k^{n-1}$. Let $\mu<\omega$.

A table of any self- $k$-al function has a line $\left(b_{1}, \ldots, b_{n} ; 0\right)$ for some $b_{i}$. The table has also a line $\left(b_{1}^{\prime}, \ldots, b_{n}^{\prime} ; l\right)$ with all $b_{i}^{\prime}$ such that $b_{i}^{\prime}=l \wedge b_{i} \neq 0$ and with all $b_{i}^{\prime}$ such that $b_{i}^{\prime} \neq l \wedge b_{i}=0$.

Let $l=0$. If all $b_{i} \neq 0$ or all $b_{j}^{\prime} \neq 0$ then $\mu=0$ otherwise $\mu=1$ since all $b_{i}=b_{i}^{\prime}=0$ are absent, i.e., a column with 2 zeros is absent but a column with 1 zero exists.

Let $l \neq 0$. Then there is an $l_{0}$ such that $l+l_{0}=0$. And there is a line $\left(b_{1}^{\prime}+l_{0}, \ldots, b_{n}^{\prime}+l_{0} ; 0\right)$ with $b_{i}^{\prime}+l_{0}=0$ if $b_{i}^{\prime} \neq b_{i}$ and with $b_{i}^{\prime}+l_{0} \neq 0$ if $b_{i}^{\prime}=b_{i}$. Then $\mu=0$ or $\mu=1$.

The theorem is proved.

We have used the relation $\rho_{1}$. But we have the same result for relations

$$
\left\{\begin{array}{ccccc}
0 & b_{1} & \ldots & b_{k-2} & b_{k-1} \\
b_{1} & b_{2} & \ldots & b_{k-1} & 0
\end{array}\right\}
$$

Lemma. A function has a Boolean projection if $\mu \neq 0$.

Proof. Let $\mu \neq 0$. Then a value of $f\left(x_{1}, . ., x_{n}\right)$ equals some of the $x_{i}$ for every function since otherwise $\mu=0$. Hence, if $x_{i} \in\{0,1\}$ then $f\left(x_{1}, \ldots, x_{n}\right) \in\{0,1\}$.

\section{Numerical Results in 3-Valued Logic}

\subsection{Classification of Self-Dual Functions}

Any classification must use disjoint classes. But any class belongs only to one clone. Therefore classification with classes and classification with clones are the same.
We call clones of self-dual functions briefly clones.

We must give the graph of inclusions of clones. The graph begins at the clone containing all other clones. Then the graph has 3 branches because there are 3 dual operators:

$$
s_{i}\left(f\left(s_{i}\left(x_{1}\right), \ldots, s_{i}\left(x_{n}\right)\right)\right)
$$

where

$$
S_{1}=\left(\begin{array}{lll}
0 & 1 & 2 \\
0 & 2 & 1
\end{array}\right), \quad S_{2}=\left(\begin{array}{lll}
0 & 1 & 2 \\
1 & 0 & 2
\end{array}\right), \quad S_{3}=\left(\begin{array}{lll}
0 & 1 & 2 \\
2 & 1 & 0
\end{array}\right)
$$

and $s_{i}^{-1}=s_{i}$.

The branches are isomorphic with respect to inclusions. Therefore we can give the graph of only one branch created by $s_{1}$. And we call clones created by $s_{1}$ just clones.

The branch begins with the clone containing all the other clones. The clone is the set of functions preserving the relation $\rho_{1}=\left\{\begin{array}{lll}0 & 1 & 2 \\ 0 & 2 & 1\end{array}\right\}$.

The inclusion graph is presented in figure 1 .

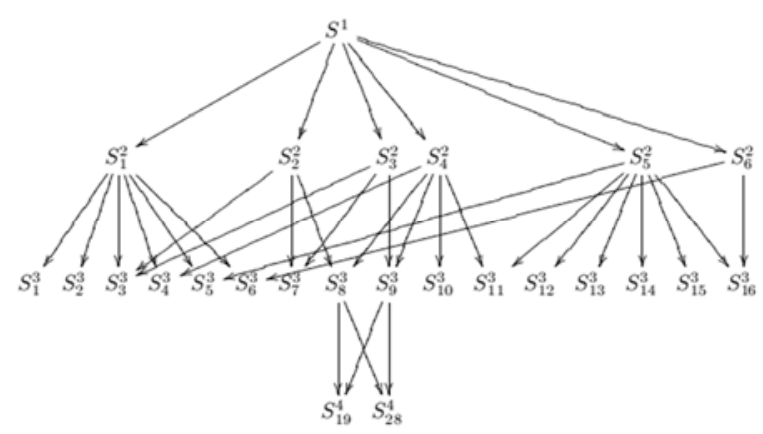

Figure 1. Three levels of inclusion graph of self-dual clones, fourth level has 39 points and web of edges. Some points (19 and 28) of forth level form non-lattice part of graph.

\subsection{Self-Dual Clones and Their Preserving Relations}

We give the set of clones and their preserved relations.

$$
\begin{aligned}
& S=\operatorname{Pol}\left\{\left\{\begin{array}{lll}
0 & 1 & 2 \\
0 & 2 & 1
\end{array}\right\}\right\} \\
& S_{1}^{2}=\operatorname{Pol}\{S,\{0\}\} \\
& S_{2}^{2}=\operatorname{Pol}\left\{S,\left\{\begin{array}{ll}
0 & 1
\end{array}\right\}\right\} \\
& S_{3}^{2}=\operatorname{Pol}\left\{S,\left\{\begin{array}{ll}
0 & 2
\end{array}\right\}\right\} \\
& S_{4}^{2}=\operatorname{Pol}\{S,\{1\}\} \\
& S_{5}^{2}=\operatorname{Pol}\left\{S,\left\{\begin{array}{ll}
1 & 2
\end{array}\right\}\right\} \\
& S_{6}^{2}=\operatorname{Pol}\left\{S,\left\{\begin{array}{lllll}
0 & 1 & 1 & 2 & 2 \\
0 & 1 & 2 & 1 & 2
\end{array}\right\}\right\}
\end{aligned}
$$




$$
\begin{aligned}
& S_{1}^{3}=\operatorname{Pol}\left\{S_{1}^{2},\left\{\begin{array}{lllll}
0 & 0 & 0 & 1 & 2 \\
0 & 1 & 2 & 0 & 0
\end{array}\right\}\right\} \\
& S_{2}^{3}=\operatorname{Pol}\left\{S_{1}^{2},\left\{\begin{array}{llllllll}
0 & 0 & 1 & 1 & 1 & 2 & 2 & 2 \\
1 & 2 & 0 & 1 & 2 & 0 & 1 & 2
\end{array}\right\}\right\} \\
& S_{3}^{3}=\operatorname{Pol}\left\{S_{1}^{2},\left\{\begin{array}{ll}
0 & 0 \\
0 & 1
\end{array}\right\}\right\}, \operatorname{Pol}\left\{S_{2}^{2},\{0\}\right\}, \operatorname{Pol}\left\{S_{3}^{2},\{0\}\right\} \\
& S_{4}^{3}=\operatorname{Pol}\left\{S_{1}^{2},\left\{\begin{array}{l}
0 \\
1
\end{array}\right\}, \operatorname{Pol}\left\{S_{4}^{2},\{0\}\right\}\right. \\
& S_{5}^{3}=\operatorname{Pol}\left\{S_{1}^{2},\left\{\begin{array}{ll}
0 & 0 \\
1 & 2
\end{array}\right\}\right\}, \operatorname{Pol}\left\{S_{5}^{2},\{0\}\right\} \\
& S_{6}^{3}=\operatorname{Pol}\left\{S_{1}^{2},\left\{\begin{array}{lllll}
0 & 1 & 1 & 2 & 2 \\
0 & 1 & 2 & 1 & 2
\end{array}\right\}\right\}, \operatorname{Pol}\left\{S_{6}^{2},\{0\}\right\} \\
& S_{7}^{3}=\operatorname{Pol}\left\{S_{2}^{2},\left\{\begin{array}{ll}
1 & 1 \\
1 & 2
\end{array}\right\}\right\}, \operatorname{Pol}\left\{S_{3}^{2},\left\{\begin{array}{ll}
1 & 1 \\
1 & 2
\end{array}\right\}\right\} \\
& S_{8}^{3}=\operatorname{Pol}\left\{S_{2}^{2},\left\{\begin{array}{l}
2 \\
1
\end{array}\right\}, \operatorname{Pol}\left\{S_{3}^{2},\left\{\begin{array}{ll}
0 & 1
\end{array}\right\}\right\}\right. \\
& S_{9}^{3}=\operatorname{Pol}\left\{S_{3}^{2},\{1\}\right\}, \operatorname{Pol}\left\{S_{4}^{2},\left\{\begin{array}{ll}
0 & 2
\end{array}\right\}\right\} \\
& S_{10}^{3}=\operatorname{Pol}\left\{S_{4}^{2},\left\{\begin{array}{llllllll}
0 & 0 & 1 & 1 & 1 & 2 & 2 & 2 \\
1 & 2 & 0 & 1 & 2 & 0 & 1 & 2
\end{array}\right\}\right\} \\
& S_{11}^{3}=\operatorname{Pol}\left\{S_{4}^{2},\left\{\begin{array}{ll}
1 & 1 \\
1 & 2
\end{array}\right\}\right\}, \operatorname{Pol}\left\{S_{5}^{2},\{1\}\right\} \\
& S_{12}^{3}=\operatorname{Pol}\left\{S_{5}^{2},\left\{\begin{array}{lllll}
0 & 0 & 0 & 1 & 2 \\
0 & 1 & 2 & 0 & 0
\end{array}\right\}\right\} \\
& S_{13}^{3}=\operatorname{Pol}\left\{S_{5}^{2},\left\{\begin{array}{lllll}
0 & 1 & 1 & 2 & 2 \\
1 & 1 & 2 & 1 & 2
\end{array}\right\}\right\} \\
& S_{14}^{3}=\operatorname{Pol}\left\{S_{5}^{2},\left\{\begin{array}{lllllll}
0 & 0 & 0 & 1 & 1 & 2 & 2 \\
0 & 1 & 2 & 1 & 2 & 1 & 2
\end{array}\right\}\right\} \\
& S_{15}^{3}=\operatorname{Pol}\left\{S_{5}^{2},\left\{\begin{array}{lllll}
0 & 1 & 1 & 2 & 2 \\
0 & 1 & 2 & 1 & 2
\end{array}\right\}, \operatorname{Pol}\left\{S_{6}^{2},\left\{\begin{array}{lll}
1 & 2
\end{array}\right\}\right\}\right. \\
& S_{16}^{3}=\operatorname{Pol}\left\{S_{5}^{2},\left\{\begin{array}{llllllll}
0 & 0 & 1 & 1 & 1 & 2 & 2 & 2 \\
1 & 2 & 0 & 1 & 2 & 0 & 1 & 2
\end{array}\right\}\right\}
\end{aligned}
$$

\subsection{Classification of Self-3-Al Functions}

The classification is presented in figure 2 by an inclusion graph.

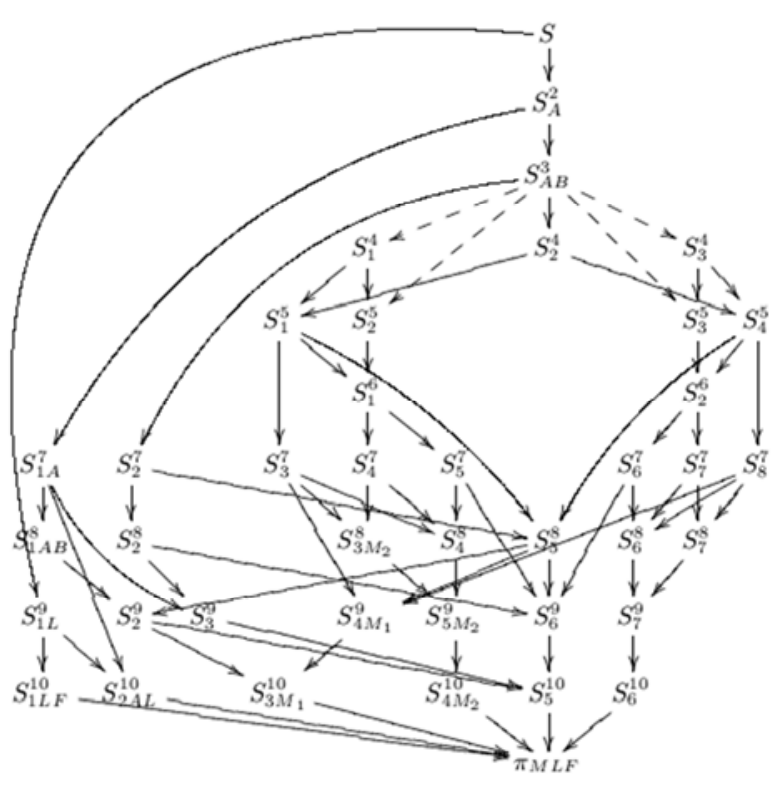

Figure 2. Inclusion graph of clones of self-3-al functions. Subscripts: $\$ A \$$ means clones of $\$$ lalpha-functions, $\$ B \$$ means clones with Boolean projections (all chains of $\$ S \_3 \$$ have clones of \$lalpha $\$$ functions with Boolean projections, implicitly), \$M\$ means clones of monotonic functions with all types of order, $\$ M_{-} 1 \$$ means clones of monotonic functions with order $\$ 1<2<0 \$, \$ M 2 \$$ means clones of monotonic functions with order $\$ 2<0<1 \$$, $\$ L \$$ means clones of linear functions. Dashed arrows mean infinite chains of clones, $\$ S^{\wedge} 3 \$$ has Boolean projection $\$ C_{-} 4 \$$ (in Post's notation), $\$ S^{\wedge} 4$ _ $1 \$$ and $\$ S^{\wedge} 4$ _ $\$$ have Boolean projections $\$ F^{\wedge}$ infty_ $1 \$$ and $\$ F^{\wedge}$ infty_5\$ respectively, $\$ S^{\wedge} 5 \_1 \$$ and $\$ S^{\wedge} 5$ _ $3 \$$ have Boolean projections $\$ F^{\wedge}$ infty_2\$ and $\$ F^{\wedge}$ infty_ $6 \$$ respectively, $\$$ |pi $\$$ is clone of projective (selective) functions.

The created graph is not a lattice as it follows from figure 3.
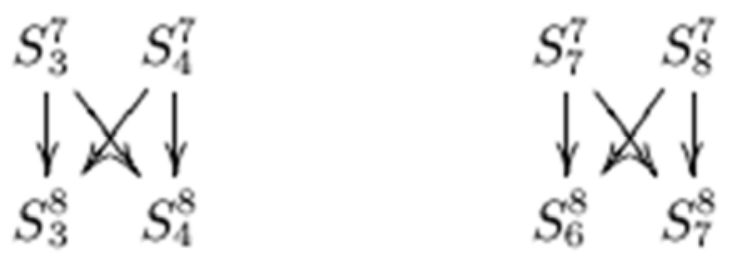

Figure 3. Non-lattice parts of inclusion graph.

\subsection{Clones of Functions Preserving Relations}

We give clones and relations preserved by functions of the clones.

$$
\begin{gathered}
\left.S=\operatorname{Pol}\left\{\begin{array}{lll}
0 & 1 & 2 \\
1 & 2 & 0
\end{array}\right\}\right\} \\
S^{2}=\operatorname{Pol}\{S,\{0\}\} \\
S^{3}=\operatorname{Pol}\left\{S^{2},\left\{\begin{array}{l}
0 \\
1
\end{array}\right\}\right\} \\
S_{1}^{4}=\operatorname{Pol}\left\{S^{3},\left\{\begin{array}{lll}
0 & 0 & 1 \\
0 & 1 & 0
\end{array}\right\}\right\}
\end{gathered}
$$




$$
\begin{aligned}
& S_{2}^{4}=\operatorname{Pol}\left\{S^{3},\left\{\begin{array}{lll}
0 & 0 & 1 \\
0 & 1 & 1
\end{array}\right\}\right\} \\
& S_{3}^{4}=\operatorname{Pol}\left\{S^{3},\left\{\begin{array}{lll}
0 & 0 & 2 \\
0 & 1 & 1
\end{array}\right\}\right\} \\
& S_{1}^{5}=\operatorname{Pol}\left\{S_{1}^{4},\left\{\begin{array}{lll}
0 & 0 & 1 \\
0 & 1 & 1
\end{array}\right\}\right\}=\operatorname{Pol}\left\{S_{2}^{4},\left\{\begin{array}{lll}
0 & 0 & 1 \\
0 & 1 & 0
\end{array}\right\}\right\} \\
& S_{2}^{5}=\operatorname{Pol}\left\{S^{3} \vee S_{1}^{4},\left\{\begin{array}{lllllll}
0 & 0 & 0 & 0 & 1 & 1 & 1 \\
0 & 0 & 1 & 1 & 0 & 0 & 1 \\
0 & 1 & 0 & 1 & 0 & 1 & 0
\end{array}\right\}\right\} \\
& S_{3}^{5}=\operatorname{Pol}\left\{S^{3},\left\{\begin{array}{lllllll}
0 & 0 & 0 & 0 & 2 & 2 & 2 \\
0 & 0 & 1 & 1 & 0 & 1 & 1 \\
0 & 1 & 0 & 1 & 1 & 0 & 1
\end{array}\right\}\right\}=\operatorname{Pol}\left\{S_{2}^{4},\left\{\begin{array}{lll}
0 & 0 & 1 \\
0 & 1 & 0
\end{array}\right\}\right\} \\
& S_{4}^{5}=\operatorname{Pol}\left\{S_{2}^{4},\left\{\begin{array}{lll}
0 & 0 & 1 \\
0 & 1 & 0
\end{array}\right\}\right\}=\operatorname{Pol}\left\{S_{3}^{4},\left\{\begin{array}{lll}
0 & 0 & 1 \\
0 & 1 & 1
\end{array}\right\}\right\} \\
& S_{1}^{6}=\operatorname{Pol}\left\{S_{1}^{5},\left\{\begin{array}{lllllll}
0 & 0 & 0 & 0 & 1 & 1 & 1 \\
0 & 0 & 1 & 1 & 0 & 0 & 1 \\
0 & 1 & 0 & 1 & 0 & 1 & 0
\end{array}\right\}\right\}=\operatorname{Pol}\left\{S_{2}^{5},\left\{\begin{array}{lll}
0 & 0 & 1 \\
0 & 1 & 1
\end{array}\right\}\right\} \\
& S_{2}^{6}=\operatorname{Pol}\left\{S_{3}^{5},\left\{\begin{array}{lll}
0 & 0 & 1 \\
0 & 1 & 1
\end{array}\right\}\right\}=\operatorname{Pol}\left\{S_{4}^{5},\left\{\begin{array}{lllllll}
0 & 0 & 0 & 0 & 2 & 2 & 2 \\
0 & 0 & 1 & 1 & 0 & 1 & 1 \\
0 & 1 & 0 & 1 & 1 & 0 & 1
\end{array}\right\}\right\} \\
& S_{1}^{7}=\operatorname{Pol}\left\{S^{2},\left\{\begin{array}{lll}
0 & 1 & 2 \\
0 & 2 & 1
\end{array}\right\}\right\} \\
& S_{2}^{7}=\operatorname{Pol}\left\{S^{3},\left\{\begin{array}{ll}
0 & 1 \\
0 & 2
\end{array}\right\}\right\} \\
& S_{3}^{7}=\operatorname{Pol}\left\{S_{1}^{5},\left\{\begin{array}{llll}
0 & 0 & 0 & 1 \\
0 & 1 & 2 & 0
\end{array}\right\}\right\} \\
& S_{4}^{7}=\operatorname{Pol}\left\{S_{1}^{6},\left\{\begin{array}{llll}
0 & 0 & 0 & 1 \\
0 & 1 & 2 & 0
\end{array}\right\}\right\} \\
& S_{5}^{7}=\operatorname{Pol}\left\{S_{1}^{6},\left\{\begin{array}{llll}
0 & 0 & 0 & 1 \\
0 & 0 & 1 & 1 \\
0 & 0 & 1 & 0
\end{array}\right\}\right\} \\
& S_{6}^{7}=\operatorname{Pol}\left\{S^{2},\left\{\begin{array}{llll}
0 & 0 & 0 & 2 \\
0 & 0 & 2 & 2 \\
0 & 1 & 1 & 0
\end{array}\right\}\right\} \\
& S_{7}^{7}=\operatorname{Pol}\left\{S^{2},\left\{\begin{array}{llll}
0 & 0 & 0 & 2 \\
0 & 1 & 2 & 0
\end{array}\right\}\right\}
\end{aligned}
$$$$
S_{8}^{7}=\operatorname{Pol}\left\{S^{2},\left\{\begin{array}{llll}
0 & 0 & 0 & 2 \\
0 & 1 & 2 & 0
\end{array}\right\}\right\}
$$$$
S_{1}^{8}=\operatorname{Pol}\left\{S_{1}^{7},\left\{\begin{array}{ll}
0 & 0 \\
0 & 1
\end{array}\right\}\right\}
$$$$
S_{2}^{8}=\operatorname{Pol}\left\{S_{2}^{7},\left\{\begin{array}{lll}
0 & 0 & 0 \\
0 & 0 & 1 \\
0 & 1 & 0
\end{array}\right\}\right\}
$$$$
S_{3}^{8}=\operatorname{Pol}\left\{S_{3}^{7} \vee S_{4}^{7},\left\{\begin{array}{lllll}
0 & 0 & 0 & 1 & 1 \\
0 & 1 & 2 & 0 & 1
\end{array}\right\}\right\}
$$$$
S_{6}^{8}=\operatorname{Pol}\left\{S_{5}^{7},\left\{\begin{array}{llll}
0 & 0 & 0 & 2 \\
0 & 1 & 2 & 0
\end{array}\right\}\right\}=\operatorname{Pol}\left\{S_{7}^{7} \vee S_{8}^{7},\left\{\begin{array}{llll}
0 & 0 & 0 & 2 \\
0 & 0 & 2 & 2 \\
0 & 1 & 1 & 0
\end{array}\right\}\right\}
$$$$
S_{7}^{8}=\operatorname{Pol}\left\{S_{7}^{7} \vee S_{8}^{7},\left\{\begin{array}{lllll}
0 & 0 & 0 & 2 & 2 \\
0 & 1 & 2 & 0 & 1
\end{array}\right\}\right\}
$$$$
S_{1}^{9}=\operatorname{Pol}\left\{S,\left\{\begin{array}{lllllllll}
0 & 0 & 0 & 1 & 1 & 1 & 2 & 2 & 2 \\
0 & 1 & 2 & 0 & 1 & 2 & 0 & 1 & 2 \\
0 & 2 & 1 & 2 & 1 & 0 & 1 & 0 & 2
\end{array}\right\}\right\}
$$$$
S_{2}^{9}=\operatorname{Pol}\left\{S_{1}^{8},\left\{\begin{array}{lll}
0 & 0 & 1 \\
0 & 1 & 0
\end{array}\right\}\right\}=\operatorname{Pol}\left\{S_{5}^{8},\left\{\begin{array}{lll}
0 & 1 & 2 \\
0 & 2 & 1
\end{array}\right\}\right\}
$$$$
S_{3}^{9}=\operatorname{Pol}\left\{S_{1}^{7},\left\{\begin{array}{lll}
0 & 0 & 1 \\
0 & 1 & 0
\end{array}\right\}\right\}=\operatorname{Pol}\left\{S_{2}^{8},\left\{\begin{array}{lll}
0 & 1 & 2 \\
0 & 2 & 1
\end{array}\right\}\right\}
$$$$
S_{4}^{9}=\operatorname{Pol}\left\{S_{3}^{7} \vee S_{8}^{7},\left\{\begin{array}{ll}
0 & 1 \\
0 & 2
\end{array}\right\}\right\}=\operatorname{Pol}\left\{S_{5}^{8},\left\{\begin{array}{llll}
0 & 0 & 0 & 1 \\
0 & 1 & 2 & 0
\end{array}\right\}\right\}
$$$$
S_{5}^{9}=\operatorname{Pol}\left\{S_{3}^{8},\left\{\begin{array}{llll}
0 & 0 & 0 & 1 \\
0 & 0 & 1 & 1 \\
0 & 1 & 0 & 1
\end{array}\right\}\right\}=\operatorname{Pol}\left\{S_{4}^{8},\left\{\begin{array}{lllll}
0 & 0 & 0 & 1 & 1 \\
0 & 1 & 2 & 0 & 1
\end{array}\right\}\right\}
$$$$
S_{6}^{9}=\operatorname{Pol}\left\{S_{5}^{7},\left\{\begin{array}{lllll}
0 & 0 & 0 & 1 & 1 \\
0 & 1 & 2 & 0 & 1
\end{array}\right\}\right\}=\operatorname{Pol}\left\{S_{6}^{7},\left\{\begin{array}{lllll}
0 & 0 & 0 & 2 & 2 \\
0 & 1 & 2 & 0 & 1
\end{array}\right\}\right\}=
$$$$
=\operatorname{Pol}\left\{S_{2}^{8},\left\{\begin{array}{lll}
0 & 0 & 0 \\
0 & 0 & 1 \\
0 & 1 & 0
\end{array}\right\}\right\}=\operatorname{Pol}\left\{S_{5}^{8},\left\{\begin{array}{lll}
0 & 0 & 1 \\
0 & 1 & 0 \\
0 & 1 & 1
\end{array}\right\}\right\}=
$$ 


$$
\begin{aligned}
& S_{7}^{9}=\operatorname{Pol}\left\{S_{6}^{8},\left\{\begin{array}{lllll}
0 & 0 & 0 & 2 & 2 \\
0 & 1 & 2 & 0 & 1
\end{array}\right\}\right\}=\operatorname{Pol}\left\{S_{7}^{8},\left\{\begin{array}{llll}
0 & 0 & 0 & 2 \\
0 & 0 & 2 & 2 \\
0 & 1 & 1 & 0
\end{array}\right\}\right\} \\
& S_{1}^{10}=\operatorname{Pol}\left\{S_{1}^{9},\left\{\begin{array}{llllll}
0 & 0 & 1 & 1 & 2 & 2 \\
0 & 1 & 1 & 2 & 0 & 2
\end{array}\right\}\right\} \\
& S_{2}^{10}=\operatorname{Pol}\left\{S_{1}^{7},\left\{\begin{array}{lll}
0 & 0 & 1 \\
0 & 1 & 0 \\
0 & 1 & 1
\end{array}\right\}\right\}=\operatorname{Pol}\left\{S_{1}^{9},\{0\}\right\} \\
& S_{3}^{10}=\operatorname{Pol}\left\{S_{2}^{9},\left\{\begin{array}{llll}
0 & 0 & 0 & 1 \\
0 & 1 & 2 & 0
\end{array}\right\}\right\}=\operatorname{Pol}\left\{S_{4}^{9},\left\{\begin{array}{lll}
0 & 1 & 2 \\
0 & 2 & 1
\end{array}\right\}\right\} \\
& S_{4}^{10}=\operatorname{Pol}\left\{S_{5}^{9},\left\{\begin{array}{lllll}
0 & 0 & 0 & 0 & 1 \\
0 & 0 & 0 & 1 & 1 \\
0 & 1 & 2 & 0 & 1
\end{array}\right\}\right\} \\
& S_{5}^{10}=\operatorname{Pol}\left\{S_{2}^{9},\left\{\begin{array}{llll}
0 & 0 & 0 & 1 \\
0 & 1 & 2 & 0
\end{array}\right\}\right\}=\operatorname{Pol}\left\{S_{3}^{9},\left\{\begin{array}{lll}
0 & 0 & 1 \\
0 & 1 & 0
\end{array}\right\}\right\}= \\
& =\operatorname{Pol}\left\{S_{6}^{9},\left\{\begin{array}{lll}
0 & 1 & 2 \\
0 & 2 & 1
\end{array}\right\}\right\} \\
& S_{6}^{10}=\operatorname{Pol}\left\{S_{7}^{9},\left\{\begin{array}{lllll}
0 & 0 & 0 & 0 & 2 \\
0 & 0 & 0 & 2 & 2 \\
0 & 1 & 2 & 0 & 2
\end{array}\right\}\right\} \\
& \pi=\operatorname{Pol}\left\{S_{1}^{10}, 0\right\}=\operatorname{Pol}\left\{S_{2}^{10},\left\{\begin{array}{ll}
0 & 0 \\
0 & 1
\end{array}\right\}\right\}=\operatorname{Pol}\left\{S_{3}^{10},\left\{\begin{array}{lll}
0 & 0 & 1 \\
0 & 1 & 2
\end{array}\right\}\right\}= \\
& =\operatorname{Pol}\left\{S_{4}^{10},\left\{\begin{array}{lll}
0 & 0 & 1 \\
0 & 1 & 2
\end{array}\right\}\right\}=\operatorname{Pol}\left\{S_{5}^{10},\left\{\begin{array}{ll}
0 & 1 \\
0 & 2
\end{array}\right\}\right\}=\operatorname{Pol}\left\{S_{6}^{10},\left\{\begin{array}{ll}
0 & 1 \\
0 & 2
\end{array}\right\}\right\}
\end{aligned}
$$

\section{Conclusion}

The main problem of any theory, including the theory of multi-valued logic, is a classification of objects of this theory.

Partial classification of clones of functions in multi-valued logic was given in the works of S. Jablonski [2] and A. Mal'cev [8].
More detailed classification of clones was given by I. Rosenberg [9]. Top level of this classification contains a clone of all functions. The next level has 6 groups of clones, but the main group contains clones of self-m-al functions. This group was named clones of self-dual functions.

In the first time, we gave full classification of the group. Number of classes of the classification is $k-1$. We named them self-m-al at $2 \leq \mathrm{m} \leq \mathrm{k}$. We built the theory of self-k-al functions and proved 10 important theorems. We get numerical results for 3-valued logic and found that inclusion graphs of clones of self-dual and of self-3-al functions are not lattices.

\section{References}

[1] Malkov M. A., Classifications of closed sets of functions in multi-valued logic, SOP transactions on applied math. 1:3, 96-105 (2014),

http://www.scipublish.com/journals/AM/papers/download/210 2-970.pdf.

[2] Yablonskiy S. V.: Functional constructions in many-valued logics (Russian). Tr. Mat. Inst. Steklova, 515-142 (1958).

[3] Lau D., Functions algebra on finite sets, Springer (2006).

[4] Csàkàny B., All minimal clones on the three-element set, Acta Cybernet., 6, 227-238 (1983).

[5] Marchenkov S. S., Demetrovics J., Hannak L., On closed classes of self-dual functions in P3. (Russian) Metody Diskretn. Anal. 34, 38-73 (1980).

[6] Machida H., On closed sets of three-valued monotone logical functions. In: Colloquia Mathematica Societatis Janos Bolyai 28, Finite Algebra and multiple-valued logic, Szeged (Hungary), 441-467 (1979).

[7] Post E. L., The two-valued iterative systems of mathematical logic. Princeton Univ. Press, Princeton (1941).

[8] Mal'cev A. I., Iterative Post algebras, NGU, Novosibirsk, (Russian) (1976).

[9] Rosenberg, I. G., Über die Verschiedenheit maximaler Klassen in Pk. Rev. Roumaine Math. Pures Appl. 14, 431-438 (1969).

[10] Malkov M. A. Classifications of Boolean Functions and Their Closed Sets, SOP transactions on applied math. 1:2, 172-193 (2014),

http://www.scipublish.com/journals/AM/papers/download/210 2-522.pdf. 